Araştırma Makalesi - Research Article

\title{
3B Yazıcı ile Elde Edilen Mandibula Modellerinin Boyutsal Değerlendirilmesi
}

\author{
Mehmet Mustafa ERDOĞAN ${ }^{1}$, Tugçe ŞİMŞEK ${ }^{2}$, Levent UĞUR ${ }^{3 *}$
}

Geliş / Received: 07/05/2020

Revize / Revised: 05/06/2020

Kabul / Accepted: 10/06/2020

$\overline{\mathbf{o z}}$

Amaç: Çalışmamızda bilgisayarlı tomografi görüntülerinden elde edilen 3B (üç boyutlu) mandibula modeli üretiminde kullanılan parametrelerden alt eşik değişimi ve katman yüksekliğinin değiştirilmesinin model boyutu üzerindeki etkisi araştırılmıştır. Metod: 35 yaşında sağlıklı bir hastanın maksillofasiyal BT görüntüleri kullanılarak tasarlanan ve basılan 3B mandibula modelinin segmentasyon işleminde eşik değeri sırasıyla 200,175 ve 150 Hounsfield birimi (HU) alınarak modeller oluşturuldu. Oluşturulan modellerin polilaktik asit (PLA) filament malzemeden baskısında $0.1,0.2$ ve $0.3 \mathrm{~mm}$ baskı katman yüksekliği kullanıldı. Elde edilen modeller yüksek çözünürlüklü tarayıcı ile taranarak STL formatında dataları bilgisayara aktarıldı. Taranan modeller referans model ile karşılaştırılarak aralarındaki fark belirlendi. Deney tasarımı olarak Taguchi L9 ortogonal dizini kullanıldı. Taguchi metodunda referans model ile meydana gelen fark için en küçük en iyi sinyal gürültü oranı denklemi kullanıldı. Sonuç: Deneyler sonucunda iki faktör arasında farka en çok etkiyi eşik değerinin yaptığı gözlemlenmiştir. Ayrıca 3B yazıcı ile elde edilen mandibula modelleri ile BT'den elde edilmiş olan anatomik model karşılaştırmasında modellerin birbirine yakın olduğu gözlemlenmiştir.

Anahtar Kelimeler- 3B Yazıcı, Mandibula, Boyutsal Doğruluk

1İletişim: mmerdogan2001@yahoo.com (https://orcid.org/0000-0001-9955-0704)

Kulak Burun Boğaz Kliniği Tıp Fakültesi, Amasya Üniversite, Merkez, Amasya

2İletişim: tugsek@hotmail.com (https://orcid.org/0000-0002-1425-8833)

Kulak Burun Boğaz Kliniği Tıp Fakültesi, Amasya Üniversite, Merkez, Amasya

3*Sorumlu yazar iletişim: leventozge@gmail.com (https://orcid.org/0000-0003-3447-3191)

Makine Mühendisliği Bölümü, Teknoloji Fakültesi, Amasya Üniversite, Merkez, Amasya 


\title{
Dimensional Evaluation of The Mandible Models With Obtained 3D Printer
}

\begin{abstract}
Objective: In our study, the effect of changing the lower threshold and layer height on the model size from the parameters used in the production of $3 \mathrm{D}$ (three-dimensional) mandible model obtained from computed tomography images was investigated. Method: We used maxillofacial CT images of a 35-year-old healthy patient in the segmentation and printing process of 3D mandible model. In the segmentation process, the threshold value was used 200,175 and 150 Hounsfield units (HU), printing layer height was $0.1,0.2$, and $0.3 \mathrm{~mm}$ in the printing of the created models of polylactic acid (PLA) filament material. The obtained models were scanned with a high precision scanner. Their data in STL format were transferred to the computer, then scanned models were compared with the reference model, and the difference between them was determined. The Taguchi L9 orthogonal index was used as the experimental design. In the Taguchi method, the smallest best signal to noise ratio equation was used for the difference with the reference model Conclusion: As a result of the experiments, it was observed that the threshold value had the most effect on the difference between the two factors. Also, it was observed that in the comparison of the mandible models obtained with a 3D printer and the anatomical model obtained from CT, the models were similar.
\end{abstract}




\section{GİRIŞ}

Beslenme, konuşma ve yüz estetiği için çok önemli fonksiyonlara sahip olan mandibula (çene kemiği) trafik kazaları, ateşli silah yaralanmaları, darp ve bazı tümöral hastalıklar veya enfeksiyon hastalıkları nedeni ile normal yapısını yitirebilir ve rekonstrükte edilme ihtiyacı doğabilir. Rekonstrüksiyonun amacı, çene ve yüz estetiğinin ve simetrisinin korunarak iyi fonksiyonel sonuçların elde edilmesi, böylece çenenin şeklinin korunması ve gelecekte olası diş rehabilitasyonuna izin vermesini sağlamaktır [1]. Rekonstrüksiyon, mandibulaya bağlı kaslar, glenoid fossadaki kondillerin şekli ve pozisyonu, mandibulanın karmaşık anatomi ve geometrisi gibi çeşitli nedenlerden dolayı cerrahlar için zorluklar oluşturmaktadır [1].

Bu zorluklar, 3 boyutlu (3B) anatomik modellerin kullanılmasıyla önemli ölçüde aşılabilir. Cerrahın görüntüleri standart bir monitörde bilgisayarlı tomografide (BT) değerlendirmesinin aksine, 3B modellerle fiziksel olarak etkileşime girerek morfolojiyi daha iyi anlaması ve uygulamalı cerrahi planlama yapması kolaylaşır [2, 3]. Tıpta 3B baskı için en zorlayıcı kullanım durumlarından biri, cerrahi planlama için hastaya özgü anatomik modellerin olușturulmasıdır[4, 5]. Bu zorluklar tıbbi görüntüleme tekniklerinin gelişmesi ile hastanın görüntülerinin kullanılması ile ortadan kaldırılmışırı [6]. Ortaya çıkan fiziksel model cerraha ameliyathaneye girmeden önce ameliyatı planlama ve hazırlık yapma firsatı sunar.

2005-2015 yılları arasında yapılan cerrahide 3B baskıyı inceleyen158 meta-analiz çalışmasında ameliyat öncesi planlama ve ameliyathanede zaman tasarrufu gibi avantajlar tanımlamış, ancak 34 çalışmada modellerin doğruluğunun tatmin edici olmadığını vurgulamıştır [7].

Üretici firmalar doğruluk açısından spesifikasyonlar sağlamasına rağmen, bunlar klinik uygulamada çoğunlukla belirsizdir. Çünkü çoğu test izosimetrik şekilli cisimler üzerinde yapılmaktadır, ancak anatomik modellerin kullanımında daha büyük boyutsal hatalar ortaya çıkabilmektedir.

3B baskı uygulamalarındaki yanlışlıklar hastaya zarar verebilecek uygunsuz tedaviye yol açabilmektedir. Cerrahlar preoperatif olarak hazırlanan kafatası veya mandibula modellerinin tedavisi planlanan hastanın tam anatomisini sunamayabileceğinin farkında olmalı ve gerekli hallerde hastanın tedavisine bilgisayarlı tomografi veya manyetik rezonans görüntülemesine dayanarak devam etmelidir. [8]. 3B baskılı dental cerrahi k1lavuzlarda bile deformasyon olabileceği bildirilmiştir [9].

$\mathrm{Bu}$ çalışmada, mandibulanın 3B modellenmesi ve üretilmesi için kullanılan parametrelerin değiştirilmesinin model üzerindeki etkisi incelenmiştir. Özellikle, segmentasyon algoritmalarında genellikle yumuşak doku- kemik sınırını belirlemek için bir dizi eşik (threshold) kullanılmaktadır. Üst eşikler genellikle kemik için maksimum Hounsfield birimi (HU) değerini aşacak şekilde ayarlanmıştır ve genellikle kemik-yumuşak doku sınırını etkilemez. HU BT'nin mucidi Godfrey Hounsfield tarafından radyodansite için tarif edilmiş bir sayısal ölçektir. Suyun değeri 0, havanın değeri -1000 ve kemiğin değeri ise yoğunluğuna göre $+300-+2000$ 'dir. Düşük eşikler kemiği yumuşak dokudan ayırır ve bu sınırların yeri ve basılan nesnenin ortaya çıkan boyutları üzerinde doğrudan bir etkiye sahip olacaktır. Ayrıca PLA filament malzemeden yapılmış 3B parçalar üzerinde farklı yazdırma parametreleri etki etmektedir. Burada önemli noktalardan biri de katman yüksekliğidir. Çalışmamızdaki amaç alt eşik değişimi ve katman yüksekliğinin model üzerindeki etkisinin incelenmesidir. Bunun için deney tasarımı olarak Taguchi L9 ortogonal dizini kullanılmıştır.

\section{MATERYAL VE METOD}

\section{A. 3D modellerin elde edilmesi}

Bu çalışmada, 35 yaşında sağlıklı bir hastanın şekil 1'de görüldüğü gibi maksillofasiyal BT görüntüleri kullanılmıştır. BT taraması GE Brightspeed 4 BT cihazıyla Amasya Üniversitesi Tıp Fakültesi Hastanesinde yapılmıştır. Protokolümüzde yüksek çözünürlüklü görüntüler oluşturmak ve yumuşak doku görüntü parazitini en aza indirmek için 0.6 mm'lik kesit kalınlığında 512x512 piksel çözünürlügü ve yumuşak rekonstrüksiyon filtreleri kullanılarak DICOM (Digital Imaging and Communications in Medicine) formatında ham veri elde edilmiştir. Tüm DICOM verileri resim arşivleme ve iletişim Sistemi'ne (PACS-Picture Archiving and Communication Systems) kaydedildi. 


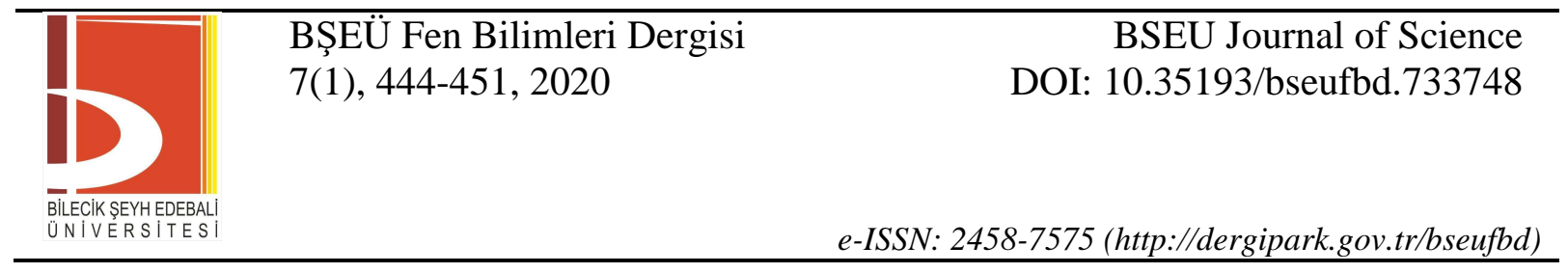

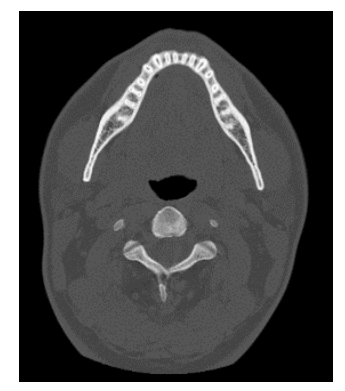

Şekil 1. Kullanılan BT'nin transverse planda görüntüsü

İnteraktif bir yazılım olan MIMICS® (Materialise's Interactive Medical Image Control System/ Materialise NV, Belgium) yazılımı kullanılarak BT görüntülerinin görselleştirme ve segmentasyon işlemleri yapıldı. Şekil 2'de görüldüğü gibi mandibula modelinin segmentasyon işleminde HU değeri sırasıyla 200, 175 ve 150 eşik değeri alınarak modeller oluşturuldu [10].

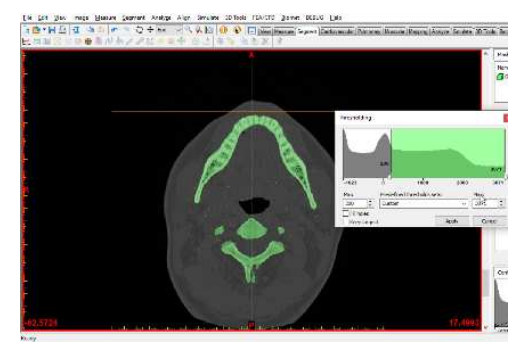

a)

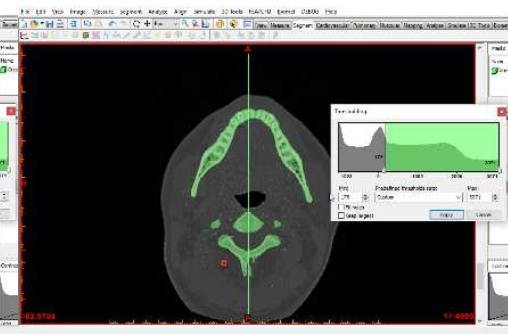

b)

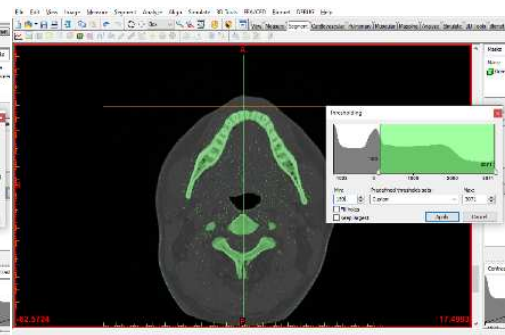

c)

Şekil 2 a) 200 HU segmentasyon görüntüsü b) 175 HU segmentasyon görüntüsü c) 150 HU segmentasyon görüntüsü

Mandibula modelinde oluşan parlama (artefakt) ve tüm yumuşak doku kalıntılarının temizlenmesi için MIMICS yazılımından geometriler tersine mühendislik yazılımı olan GEOMAGIC® Studio (Raindrop Inc., USA) programına STL (stereolithography) formatına dönüştürülerek gönderilmiştir. Herbir segmentasyon için ayrı ayrı modeller elde edilmiştir [11]. Şekil 3'de bu modellerden biri gösterilmiştir.

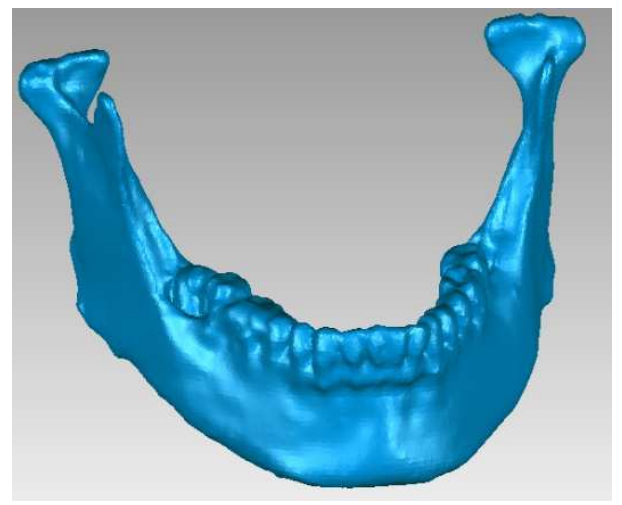

Şekil 3. Mandibula modelinin Geomagıc Studio programında düzenlenmesi. 


\section{B. Modellerin 3B Yazıcıdan elde edilmesi}

Elde edilen mandibula modelleri Şekil 4'te gösterildiği şekilde IdeaMaker 3.1.7 programı ile 3B yazıcı yazdırma tablasına konumlandırılmıştır. 3B yazıcılarda farklı yazdırma parametreleri vardır. Bunlardan biri olan katman yüksekliğinin değiştirilmesi ile modeller çift ekstrüdere sahip Raise3D Pro2 makinesinde üretilmiştir. Bu yazıcılar, termoplastik bir polyester olan Polilaktik Asit (PLA) kullanır. Tüm modellerde işlem ayarları standartlaştırılmış ve ekstrüder sıcaklığı $215^{\circ} \mathrm{C}$, oda sıcaklığı $24{ }^{\circ} \mathrm{C}$, destek $\% 20$, destek olmadan maksimum çıkıntı \%60 olarak alınmıştır.
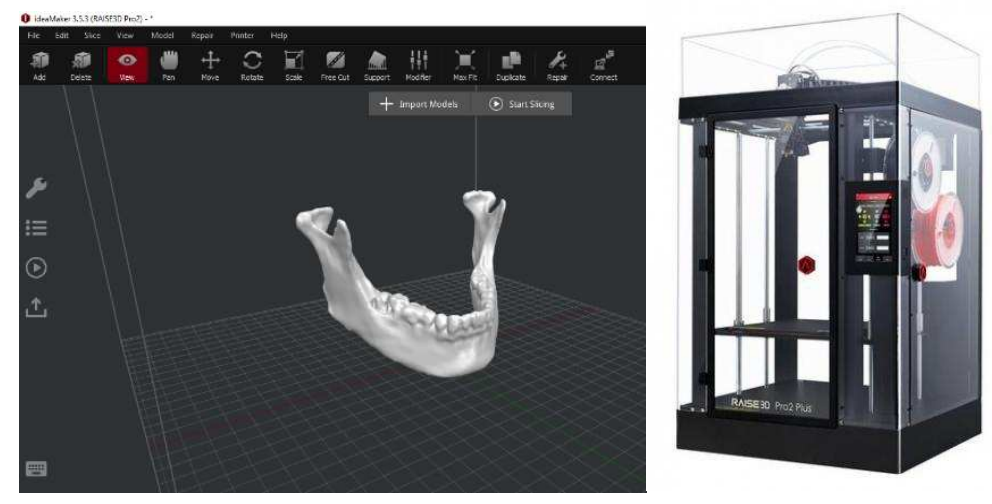

Şekil 4. Mandibula modelinin 3B yazıcı ile elde edilmesi.

\section{Taguchi Yöntemi ile Sonuçların Değerlendirilmesi}

Literatürden elde edilen veriler ile tablo 1'de modellerin tasarımında ve üretiminde kullanılan parametreler verilmiştir [11].

Tablo 1. Modellerin tasarımında ve üretiminde kullanılan parametreler ve seviye değerleri

\begin{tabular}{llll}
\hline Tasarım ve Üretim parametreleri & Seviye 1 & Seviye 2 & Seviye 3 \\
\hline Eşik Değeri (HU) & 200 & 175 & 150 \\
\hline Katman Yüksekliği (mm) & 0,1 & 0,2 & 0,3 \\
\hline
\end{tabular}

Modellemede ve üretimde kullanılan parametreler ve seviyeler tablo 2'de gösterildiği gibi Taguchi metodu ile L9 ortogonal dizilim oluşturulmuştur. Tablo 1'de verilen değere göre anatomik model ile farklar ölçülmüştür. Taguchi metodunda referans model ile meydana gelen fark için gerekli olan parametreler için, en küçük en iyi sinyal gürültü oranı denklemi kullanılarak yapılmıştır. Minitab 17 programında en küçük en iyi denklemi kullanılarak S/N oranları, seviye değerleri hesaplanmıştır. Bu denklemin sonucuna göre elde edilen S/N oranları Tablo 2'te verilmiştir. Denklemde "n" tekrarlama sayısı, "i" ve "y" ise değişkenin ölçülen değeridir.

$$
\frac{S}{N}=-10 \log \left[\frac{1}{n} \sum_{i=1}^{n} y_{i}^{2}\right]
$$


Tablo 2. L9 ortogonal dizilim kullanarak oluşturulan

\begin{tabular}{lcccc}
\hline Deney No & Eşik Değeri $(\mathbf{H U})$ & Katman Yüksekliği $(\mathbf{m m})$ & $\mathbf{R M S}(\mathbf{m m})$ & $\mathbf{S} / \mathbf{N}(\boldsymbol{d B})$ \\
\hline $\mathbf{1}$ & 150 & 0,1 & 0,215 & 13,3512 \\
\hline $\mathbf{2}$ & 150 & 0,2 & 0,271 & 11,3406 \\
\hline $\mathbf{3}$ & 150 & 0,3 & 0,292 & 10,6923 \\
\hline $\mathbf{4}$ & 175 & 0,1 & 0,126 & 17,9926 \\
\hline $\mathbf{5}$ & 175 & 0,2 & 0,178 & 14,9916 \\
\hline $\mathbf{6}$ & 175 & 0,3 & 0,195 & 14,1993 \\
\hline $\mathbf{7}$ & 200 & 0,1 & 0,124 & 18,1316 \\
\hline $\mathbf{8}$ & 200 & 0,2 & 0,156 & 16,1375 \\
\hline $\mathbf{9}$ & 200 & 0,3 & 0,174 & 15,1890 \\
\hline
\end{tabular}

\section{Modellerin Doğrulanması}

Elde edilen modeller yüksek çözünürlüklü tarayıcı (Artec Space Spider,) ile taranarak STL formatında dataları bilgisayara aktarıldı. Elde edilen modellerin her biri 3D analiz için Geomagic Studio programına gönderilerek en uygun hizalama yöntemi kullanılarak referans mandibula modeli üzerine bindirildi. Daha sonra şekil 5'de gösterildiği gibi taranan modeller referans model ile karşılaştırılarak fark noktaları ve ortalama karekök (the root mean square value (RMS)) değeri belirlendi.

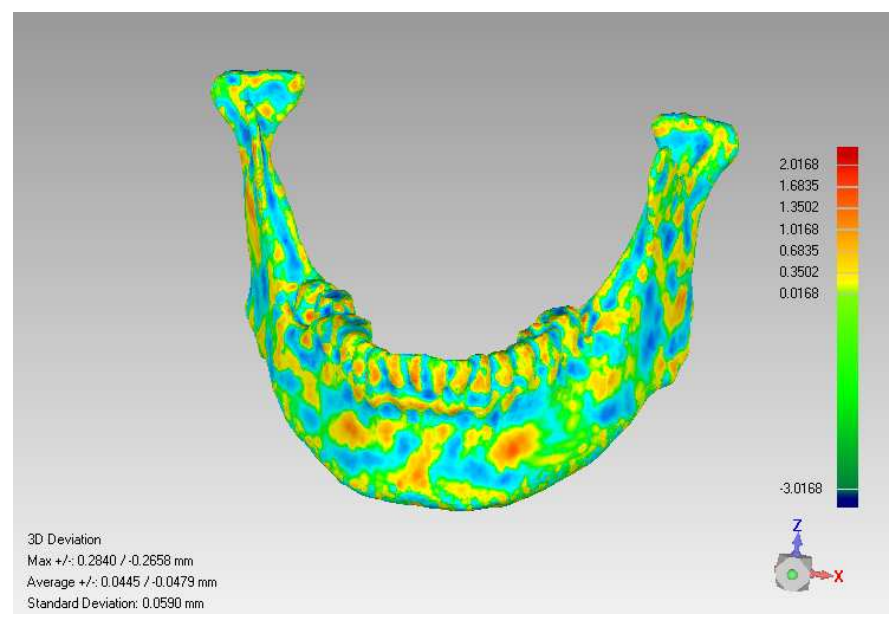

Şekil 5. Modellerin karşılaştırılması

\section{BULGULAR}

Taguchi metodu L9 ortogonal dizilim yöntemine göre oluşturulan modeller referans model ile kıyaslanarak elde edilen RMS değerleri tablo 2'de gösterilmiştir. Elde edilen verilerin analizleri için Taguchi metodunun en temel kriterleri olan sinyal/gürültü $(\mathrm{S} / \mathrm{N})$ oranı kullanılmıştır. Tablo 2'de referans modele en yakın 31 dizilimi yani, $200 \mathrm{HU}$ ve $0,1 \mathrm{~mm}$ katman kalınlığında üretilmiş modelde ulaşıldığ 1 görülmüştür. Analizler sonucunda elde edilen parametre seviyelerinin S/N oranları Tablo 3 'te verilmiştir. Bu tabloda parametre seviyeleri için en büyük ve en küçük $\mathrm{S} / \mathrm{N}$ oranı arasındaki fark gösterilmiştir. "Delta" satırındaki değeri en büyük olan parametre bu işlem için en önemli parametreyi göstermektedir. Buna göre eşik değeri en önemli parametredir. Şekil 6' da S/N oranları grafiği verilmiştir. 
Tablo 3. RMS için S/N cevap tablosu

\begin{tabular}{lcc}
\hline Level & Eşik Değeri (HU) & $\begin{array}{c}\text { Katman Yüksekliği } \\
(\mathbf{m m})\end{array}$ \\
\hline $\mathbf{1}$ & 11,79 & 16,49 \\
\hline $\mathbf{2}$ & 15,73 & 14,16 \\
\hline $\mathbf{3}$ & 16,49 & 13,36 \\
\hline Delta & 4,69 & 3,13 \\
\hline Rank & 1 & 2 \\
\hline
\end{tabular}

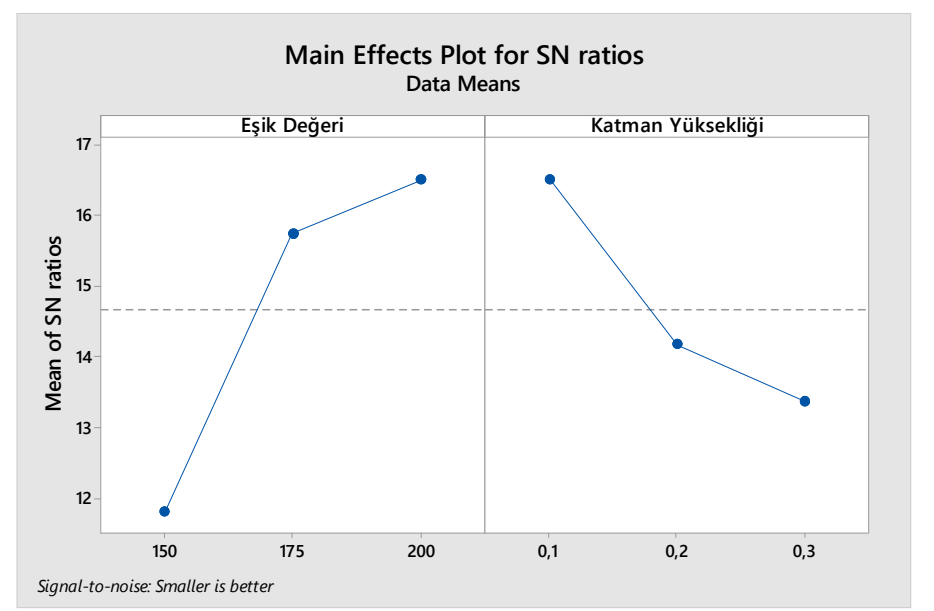

Şekil 6. RMS'nin S/N oranına göre Eşik değeri ve Katman yüksekliği faktör seviyelerinin grafiği

\section{TARTIŞMA VE SONUÇ}

3B yazıcılar tıpta cerrahi öncesi planlamada, kişiye özel anatomik model üretiminde, kişiye özel cerrahi alet veya protez üretiminde, hasta ve doktor eğitiminde, delme kılavuzları üretiminde ve doku mühendisliğinde kullanılmaktadır.[12, 13]Tıbbi uygulamalarda 3B baskının en önemli unsuru referans modele en yakın geometrinin elde edilmesidir. Boyutsal hassasiyeti zayıf modellerin üretilmesi yanlış varsayımlara yol açabilir ve en kötü durumda hastaya zarar verebilir. Hassasiyet daha önceki çalışmalarda milimetre cinsinden ifade edilmiştir [14, 15]. Bu hassasiyet pozitif veya negatif sapmalar olarak ortaya çıkabilmektedir. Bu çalışmada hassasiyet için diş hekimliği alanında yapılan diğer çalışmalarda olduğu gibi tüm değerlerin karesi olan RMS değeri baz alınmıştır[16].

Çalışmamızda 3B mandibula modellemesi için kullanılan parametrelerden alt eşik değişimi ve 3B yazıcı ile üretilmesinde katman yüksekliği parametrelerinin üç farklı özelliği kullanılarak 3B baskı teknolojisiyle üretilen anatomik mandibula modelleri boyutsal doğrulukları açısından karşılaştırılmışıtır. En iyi RMS değeri 0,124 mm olarak eşik değeri $200 \mathrm{HU}$ 'da ve $0.1 \mathrm{~mm}$ kesit kalınlığında elde edilmiştir.

Literatürde 3B yazıcı ile elde edilen modellerde referans verileri arasında genellikle $1 \mathrm{~mm}$ 'den küçük hatalar olduğu görülmüştür [17]. Bu da elde ettiğimiz sonuçların literatürle benzerlik gösterdiğini kanıtlamaktadır. Genel olarak, mandibulanın segmentasyonu için alt eşik değerinin azaltılması ile elde edilen mandibula modellerinin boyutlarının arttığını ve referans model ile aralarında önemli ölçüde farklıkların meydana geldiği gözlemlenmiştir. Ayrıca eşik değerinin düşürülmesi sonrası oluşan değişim nedeniyle 3B modeli manuel olarak onarmak gerektiğinden ek zaman ve çaba gerekmektedir. Bunun yanı sıra katman yüksekliğinin artırılmasının yine referans modele göre farklıklar oluşturduğu gözlemlenmiştir. 


\section{KAYNAKLAR}

[1] Cohen, A., et al., Mandibular reconstruction using stereolithographic 3-dimensional printing modeling technology. Oral Surgery, Oral Medicine, Oral Pathology, Oral Radiology, and Endodontology, 2009. 108(5): p. 661-666.

[2] Odeh, M., et al., Methods for verification of 3D printed anatomic model accuracy using cardiac models as an example. $3 D$ printing in medicine, 2019. 5(1): p. 6.

[3] Petzold, R., H.-F. Zeilhofer, and W. Kalender, Rapid prototyping technology in medicine-basics and applications. Computerized Medical Imaging and Graphics, 1999. 23(5): p. 277-284.

[4] Matsumoto, J.S., et al., Three-dimensional physical modeling: applications and experience at Mayo Clinic. Radiographics, 2015. 35(7): p. 1989-2006.

[5] Bastawrous, S., et al., Principles of three-dimensional printing and clinical applications within the abdomen and pelvis. Abdominal Radiology, 2018. 43(10): p. 2809-2822.

[6] Ripley, B., et al., 3D printing from MRI data: harnessing strengths and minimizing weaknesses. Journal of Magnetic Resonance Imaging, 2017. 45(3): p. 635-645.

[7] Martelli, N., et al., Advantages and disadvantages of 3-dimensional printing in surgery: a systematic review. Surgery, 2016. 159(6): p. 1485-1500.

[8] Fasel, J.H., et al., A critical inventory of preoperative skull replicas. The Annals of The Royal College of Surgeons of England, 2013. 95(6): p. 401-404.

[9] Stumpel, L.J., Deformation of stereolithographically produced surgical guides: an observational case series report. Clinical implant dentistry and related research, 2012. 14(3): p. 442-453.

[10] Ogden, K., et al. Dimensional accuracy of 3D printed vertebra. in Medical Imaging 2014: Image-Guided Procedures, Robotic Interventions, and Modeling. 2014. International Society for Optics and Photonics.

[11] Brouwers, L., et al., Validation study of 3D-printed anatomical models using 2 PLA printers for preoperative planning in trauma surgery, a human cadaver study. European Journal of Trauma and Emergency Surgery, 2019. 45(6): p. 1013-1020.

[12] Kaye, R., et al., Three dimensional printing: A review on the utility within medicine and otolaryngology. International Journal of Pediatric Otorhinolaryngology, 2016. 89: p. 145-148.

[13] Aimar, A., A. Palermo, and B. Innocenti, The role of 3D printing in medical applications: a state of the art. Journal of healthcare engineering, 2019. 2019.

[14] Silva, D.N., et al., Dimensional error in selective laser sintering and 3D-printing of models for craniomaxillary anatomy reconstruction. Journal of cranio-maxillofacial surgery, 2008. 36(8): p. 443-449.

[15] Ibrahim, D., et al., Dimensional error of selective laser sintering, three-dimensional printing and PolyJet ${ }^{\mathrm{TM}}$ models in the reproduction of mandibular anatomy. Journal of Cranio-Maxillofacial Surgery, 2009. 37(3): p. 167-173.

[16] Kim, S.-Y., et al., Precision and trueness of dental models manufactured with different 3-dimensional printing techniques. American Journal of Orthodontics and Dentofacial Orthopedics, 2018. 153(1): p. 144-153.

[17] George, E., et al., Measuring and establishing the accuracy and reproducibility of 3D printed medical models. Radiographics, 2017. 37(5): p. 1424-1450. 